\title{
Relación entre dependencia emocional y maltrato psicológico en forma de victimización y agresión en jóvenes
}

\author{
Beatriz Martín y María de la Villa Moral* \\ Universidad de Oviedo
}

- Recibido: 30 - 09 - 2018 . Aceptado: 12 - 03 - 2019 . Avance Online: 03 - 05 - 2019

RESUMEN: Antecedentes: en la literatura especializada se ha estudiado la relación de la dependencia emocional con la violencia en el noviazgo en general, pero apenas con el maltrato psicológico. El objetivo del presente trabajo es estudiar la relación entre la dependencia emocional y el maltrato psicológico en forma de victimización y agresión. Método: la muestra está compuesta por 396 adolescentes y jóvenes españoles de la población general con edades comprendidas entre 15 y 30 años $(M=19.36, D T=2.60)$. La dependencia emocional se evaluó a través del Inventario de Relaciones Interpersonales y Dependencias Sentimentales (IRIDS-100) y la victimización o agresión psicológica con el Cuestionario de Violencia entre Novios Víctima Agresor (CUVINO-VA). Resultados: los jóvenes victimizados psicológicamente y los agresores de este tipo de violencia tienen medias más altas en dependencia emocional. La dependencia emocional no se relaciona con el género, la edad ni el nivel de estudios, mientras que el maltrato psicológico sí mantiene relación con la edad y el nivel de estudios. Conclusiones: existe relación entre la dependencia emocional y el maltrato psicológico, siendo el macrofactor Autoengaño el más explicativo de esta relación. Es necesaria una mejor educación afectiva y la promoción de investigaciones sobre la dependencia emocional dada su trascendencia en la violencia en el noviazgo.

PALABRAS CLAVE: Dependencia emocional, Maltrato psicológico, Autoengaño, Victimización psicológica, Agresión psicológica.

\section{Relationship between emotional dependence and psychological abuse in the form of victimization and aggression in young people}

ABSTRACT: Background: on the specialized literature it has been studied the relationship between emotional dependence and dating violence in general, but not together with psychological abuse. The aim of this paper is to study the relationship between emotional dependence and psychological abuse in the form of victimization and aggression. Method: the sample consisted of 396 Spanish adolescents and young people from the general population aged between 15 and 30 years old $(M=19.36, D T=2.60)$. Emotional dependence was evaluated through the Inventory of Interpersonal Relationships and Sentimental Dependencies (IRIDS-100) and the victimization or psychological aggression with the Dating Violence Questionnaire Victim Aggressor (CUVINO-VA). Results: the psychologically victimized youth and the aggressors of this type of violence have higher means of emotional dependence. Emotional dependence is not related to gender, age or level of education, while psychological abuse does have a relationship with age and educational level. Conclusions: there is a relationship between emotional dependence and psychological abuse, with the macro-factor Self-deception being the most explanatory of this relationship. It is necessary a better affective education and the promotion of research on emotional dependence given its transcendence in dating violence.

KEYWORDS: Emotional dependence, Psychological abuse, Self-deception, Psychological victimization, Psychological aggression.

La Dependencia emocional se describe como un patrón crónico de demandas afectivas

\footnotetext{
*Correspondence: María de la Villa Moral.

Universidad de Oviedo

CP: 33003, Oviedo, España.

E-mail:mvilla@uniovi.es
}

(C) 2019 Sociedad Universitaria de Investigación en Psicología y Salud. Publicado por Consejo General de Colegios Oficiales de Psicólogos, España. Este es un artículo Open Access
bajo la CC BY-NC-ND licencia (http://creativecommons.org/licencias/by-nc-nd/4.0/). frustradas que tratan de satisfacerse mediante relaciones interpersonales de apego patológico (Sirvent y Moral, 2018). La identificación de sus manifestaciones patognomónicas resulta complicada no solo por la dificultad del individuo para reconocer su problemática, sino por la propia aceptación social. En relación con esta aceptación se encuentra el llamado mito del 
amor romántico que incluye creencias relativas a la equivalencia, la exclusividad, la media naranja, la fidelidad, la perdurabilidad, etc. (Barrón, De Paúl, Martínez-Iñigo y Yela, 1999). Estas ideas conllevan que en la sociedad actual se disfrace la realidad amorosa bajo un imaginario colectivo perpetuador de tales mitos mediante el que se tiende a trivializar dichas patologías, percibiéndolas como un mal de amores, pero no como un auténtico trastorno.

En los últimos años, ha sido investigada la relación de la dependencia emocional con variables de interés tales como el género (Moral y Sirvent, 2009). Así, Moral, García, Cuetos y Sirvent (2017), al comprobar las relaciones existentes entre Violencia del noviazgo, Dependencia emocional y Autoestima en adolescentes y jóvenes, obtuvieron que los varones ejercían con más frecuencia procesos de acomodación y de manipulación, hallándose un tasa superior de violencia y dependencia emocional en los estudiantes de Educación Secundaria frente a los universitarios. También se ha investigado la relación de la dependencia emocional con el perfil cognitivo (Lemos-Hoyos, Jaller-Jaramillo, González-Calle, Díaz-León, y De la Ossa, 2012), la formación de esquemas cognitivos desadaptativos (Urbiola y Estévez, 2015), el uso de las redes sociales y las nuevas tecnologías (Estévez, Urbiola, Iruarrizaga, Onaindia y Jauregui, 2017), las relaciones maritales (Kemer, Yildiz, y Bulgan, 2016), la ansiedad y la depresión (Urbiola, Estévez, Iruarrizaga, y Jáuregui, 2017) o el estilo de apego adulto (Valle y Moral, 2018), entre otros. Asimismo, resulta de interés la vinculación de la dependencia emocional con la búsqueda de sensaciones y el autoengaño, como principales factores descriptivos. Por un lado, se ha constatado que la juventud es una etapa que se caracteriza por una mayor frecuencia de comportamientos impulsivos, sobre todo en situaciones sociales cargadas emocionalmente (Gil-Olarte, Cavalcante, Paramio, Zayas, y Guil, 2017). Por otro lado, respecto al autoengaño (Self-deception) puede ser visto como una presentación no deliberada del yo para evitar la detección pública del engaño con componentes como la minimización, la negación o el pensamiento fantaseado como parte sustancial (Sirvent, Herrero, Moral y Rodríguez, 2019), desvinculándose, por tanto, del estudio de la disimulación como manejo de la impresión (Impression management) más propia del ámbito jurídico-forense (Arce, Fariña, Seijo, y Novo, 2015).

Se tiende a relacionar la dependencia emocional con la violencia en la pareja bajo la hipótesis de que podría ser uno de los elementos determinantes de la perpetuación de relaciones violentas (Amor y Echeburúa, 2010). Cabe señalar que se ha analizado qué papel juega la dependencia emocional en las víctimas (Moral et al., 2017) y, en menor medida, en los agresores (Petruccelli et al., 2014), encontrando en ambos casos una relación positiva entre los constructos. La lógica de estos estudios se enmarca en que el dependiente emocional es una persona vulnerable emocionalmente que se encuentra eclipsado por el sujeto del que depende como consecuencia de un cúmulo de ilusiones, atribuciones, procesos hedónicos y expectativas infundadas. Precisamente esta idea de perdonar todo, junto con las características ya descritas, convierten al dependiente emocional en una potencial víctima de violencia por parte de su pareja y quizá también en un potencial agresor.

Tanto en las primeras relaciones como en las parejas más consolidadas se manifiestan conductas violentas en mayor o menor medida (Batiza, 2017; Medrano, Miranda, y Figueras, 2017; Redding, Ruiz, Fernández, y Guijarro, 2017; Siödin, Wallinius, Billstedt, Hofvander, y Nilsson, 2017). En este sentido, Johnson, Giordano, Manning y Longmore (2015) han hallado que el pico de la violencia se da a los 20 años y disminuye a partir de esta edad. A su vez, López-Cepero, Rodríguez-Franco, RodríguezDíaz, Bringas y Paíno (2015) han demostrado que en las relaciones entre adolescentes y adultos jóvenes hay una serie de características propias, no aplicables a parejas casadas. Una de estas características propias es la estudiada bidireccionalidad (Rubio-Garay, López-González, Saúl, y Sánchez-Elvira-Paniagua, 2012; ZamoraDamián, Alvídrez, Aizpitarte, y Rojas-Solís, 2018), lo cual supone, en el caso de una pareja heterosexual, que el chico no es el perpetrador de la violencia y la chica la víctima, sino que ambos son agresores, aunque no del mismo tipo de violencia. De hecho, Moral et al. (2017) 
comprobaron que en las relaciones de noviazgo las chicas inician y perpetran la violencia con más frecuencia que los chicos, excepto en agresiones físicas severas. De este modo, la violencia en el noviazgo se refiere a aquellos comportamientos que, dentro de una relación íntima o de pareja, tienden a causar daño físico, psíquico y/o sexual a los miembros de la misma (Celis y Rojas, 2015), constatándose una manifiesta violencia de pareja íntima en el colectivo de entre 15 y 30 años (Jennings et al., 2017). En este sentido, los resultados son un poco inconsistentes ya que si bien se ha sugerido que a menor edad existiría una mayor prevalencia de violencia en las relaciones de noviazgo (Bonomi et al., 2012; Palmetto, Davidson, Breitbart y Rickert, 2013), en otros estudios se estima que el aumento de edad se asocia a una menor violencia (Redondo, Inglés, y García, 2017) e incluso no se han hallado relaciones estadísticamente significativas entre la violencia cometida o sufrida con la edad de los participantes (Zamora et al., 2018).

Un fenómeno observado en lo que se refiere a las relaciones de maltrato es la disociación entre la victimización de violencia por parte de la pareja y la auto-percepción de la víctima. Así, por ejemplo, Rodríguez-Franco, Antuña-Bellerín, López-Cepero, Rodríguez-Díaz y Bringas (2012) describieron un alto porcentaje de víctimas sin percepción de abuso en mujeres adolescentes y jóvenes. Es difícil establecer la prevalencia de la realidad que nos ocupa, de manera que RubioGaray, López-González, Carrasco y Amor (2017) realizaron una revisión sistemática de estudios sobre la prevalencia de esta problemática, cometida y sufrida por adolescentes y jóvenes, encontrando una gran variabilidad en los datos.

La violencia en el noviazgo se ha clasificado tradicionalmente en física, psicológica y sexual, siendo el maltrato físico la forma más fácilmente identificable, al contrario que la violencia psicológica que se considera que afecta menos a la salud de la víctima. El abuso psicológico es el más prevalente, especialmente en parejas jóvenes (Muñoz-Rivas, Graña, y González, 2011 ), el más persistente en el tiempo (Momeñe, Jáuregui, y Estévez, 2017) y tiene graves consecuencias a largo plazo como la disminución de la autoestima, así como manifestaciones psicopatológicas como la ansiedad y la depresión (Temple et al., 2016).
En virtud de lo expuesto con anterioridad, se justifica el estudio de la relación entre la dependencia emocional y la victimización o perpetración de maltrato psicológico en la pareja. En este sentido, se ha constatado que el abuso psicológico y la dependencia emocional correlacionan positivamente, lo que sugiere que a medida que aumenta el abuso psicológico, mayor será la dependencia emocional hacia la pareja (Momeñe et al., 2017).

\section{• OBJETIVOS E HIPÓTESIS}

En este estudio se plantea como objetivo analizar la relación entre la dependencia emocional y el maltrato psicológico (como víctima y como agresor) en población juvenil. Como objetivos secundarios se proponen: a) estudiar la prevalencia de estos dos constructos de forma aislada; b) comprobar si hay diferencias en la dependencia emocional y el maltrato psicológico de manera independiente, en función de la edad, el género y el nivel de estudios; y finalmente, c) analizar si la dependencia emocional guarda relación con la conciencia de maltrato, es decir, si quien es maltratado lo reconoce y qué papel juega la dependencia emocional en este proceso.

En base a la literatura publicada hasta la fecha se parte de las siguientes hipótesis: a) se observará una relación entre la dependencia emocional y el maltrato psicológico; b) existirán diferencias inter-género en dependencia emocional, puntuando los hombres más alto en Búsqueda de Sensaciones y las mujeres en Autoengaño; c) habrá un mayor número de mujeres victimizadas, aunque con una tendencia a la bidireccionalidad; d) el mayor número de casos de victimización se dará en torno a los 20 años $y$, asimismo, e) se prevé que un alto porcentaje de los victimizados no se identificará como maltratado.

\section{MÉTODO}

\section{-PARTICIPANTES}

La muestra está compuesta por 396 adolescentes y jóvenes de entre 15 y 30 años, siendo la media de edad de 19.36 años (DT $=2.60)$. Participaron 262 mujeres (66.16\%) y 
134 hombres (33.84\%) reclutados mediante muestreo incidental en varios Institutos de Asturias y Cantabria, así como en diversos Grados de Ciencias de la Salud y JurídicoSociales de la Universidad de Oviedo y en un Colegio Mayor de la misma Universidad. Esta selección de estudios puede dar cuenta de la desigual distribución de género, pues se trata de Grados y Ciclos formativos en los que hay una mayor proporción de mujeres.

En cuanto a las relaciones afectivas de la muestra, los hombres señalaron una duración media de éstas de 13.70 meses $(D T=15.86)$ y las mujeres de 20.27 meses $(D T=18.67)$. Los hombres tenían parejas o exparejas con una media de edad de 18.60 años (DT $=3.76)$, situándose más de la mitad (60.45\%) en el rango de edad 15-18 (26.12\% en rango 19-21 y $13.43 \%$ en rango $22-30)$. La media de edad de las parejas o exparejas de las mujeres fue 21.48 (DT $=4.29$ ), de las cuales un $25.95 \%$ se situaba en el rango de edad 15-18, un $34.73 \%$ en $19-21$ y un $39.31 \%$ en $22-30$. Con respecto a su nivel de estudios, el $24.63 \%$ de las parejas o exparejas de los hombres y el $14.12 \%$ de las de las mujeres tenía estudios de Educación Secundaria, el $32.09 \%$ de los hombres y el $18.32 \%$ de las mujeres tenían parejas o exparejas estudiantes de Bachiller, el 5.22\% de las parejas o exparejas de los hombres y el $19.08 \%$ de las de las mujeres cursaba o había cursado Formación profesional y el $38.06 \%$ de los hombres y el $45.42 \%$ de las mujeres tenían - habían tenido una relación afectiva con estudiantes universitarios.

\section{-INSTRUMENTOS}

Para la medición de la presencia o ausencia de dependencia emocional y sus niveles, se utilizó el Inventario de Relaciones Interpersonales y Dependencias Sentimentales (IRIDS-100) (Sirvent y Moral, 2018). Se trata de un cuestionario para medir Dependencias Relacionales compuesto por tres subescalas: Dependencia emocional (DE), Bidependencia (BDP) y Codependencia (CDP). La estructura factorial del inventario presenta siete dimensiones o macrofactores (Triada Dependiente, Acomodación, Autoengaño, Sentimientos Negativos, Identidad/Caracterosis,
Antecedentes Personales y Triada Codependiente) y 23 factores sindrómicos (Sirvent y Moral, 2018). Consta de 100 ítems evaluados mediante escala tipo Likert de cinco puntos, evaluados desde "muy en desacuerdo" a "muy de acuerdo". En este estudio se administraron los79 ítems correspondientes a la evaluación de la dependencia emocional. El alfa global de la escala es de .96 (Sirvent y Moral, 2018), siendo el valor en este estudio de .95. A su vez, la escala de Dependencia Afectiva presentó buena consistencia interna $(\alpha=.87)$, así como los macrofactores Triada Dependiente, Acomodación, Autoengaño, Sentimientos Negativos e Identidad Caracteriosis $(\alpha=$ $.86, .75, .89, .89$ y .82 respectivamente). Además, la escala correlacionó positiva y significativamente, ps $<.001$, con todos los macrofactores de la prueba.

La evaluación del maltrato psicológico se realizó con el Cuestionario de Violencia de Novios Víctima Agresor (CUVINO-VA) validado por loa autores de la escala a partir del CUVINO-R O DVQ-R (RodríguezDíaz et al., 2017). Este cuestionario evalúa comportamientos que son constitutivos de violencia en relaciones de noviazgo evaluando agresión y victimización propia y del otro, en relaciones de noviazgo, a través de dos escalas (Victimización y Agresión) de 20 ítems cada una y cinco factores (Coerción, Desapego, Humillación, Físico y Sexual). Los ítems son de respuesta tipo Likert, siendo 1 nunca y 5 casi siempre. El alfa de Cronbach de la escala Victimización es de .85 y en el Análisis factorial confirmatorio el CFI de la escala de Victimización ha sido .99 y el de Agresión .98. En este estudio el alfa de Cronbach tuvo un valor de .83 para la escala de Victimización y de .72 para la de Agresión. A su vez, la distinción entre victimizados y no victimizados se exploró en base a dos criterios distintos: la percepción del sujeto sobre su experiencia de victimización (representado por el ítem dicotómico "żTe has sentido maltratado/a?" del CUVINO-VA) y un criterio de Tolerancia cero (se considera que cualquier sujeto que responda afirmativamente a, por lo menos un ítem de los factores Humillación, Desapego o Coerción, ha sido victimizado psicológicamente). 


\section{- PROCEDIMIENTO Y ANÁLISIS DE DATOS}

Se empleó un diseño no experimental de tipo transversal a nivel descriptivo para valorar la dependencia emocional, victimización y agresión, y a nivel correlacional para establecer las relaciones entre las variables de interés.

Dado que se trata de un estudio con humanos, se obtuvo el consentimiento informado de los participantes, de acuerdo con lo que establecen los "Principios éticos para las investigaciones con humanos" de la Declaración de Helsinki. $\mathrm{Al}$ incluirse participantes menores de 18 años la realización del estudio fue aprobada por el equipo directivo de los centros y fue necesario solicitar el consentimiento de los tutores. La administración de los cuestionarios se llevó a cabo de manera grupal, ocupando veinte minutos de las clases cedidas por los profesores. Todos los participantes fueron informados del uso de los datos recabados para fines exclusivamente de investigación.

Los datos recabados fueron analizados estadísticamente con el software SPSS (IBM) versión 20. Se utilizaron estadísticos descriptivos para la muestra y las pruebas KolmogorvSmirnov y Levene para verificar la normalidad y la homocedasticidad de las variables dependientes. Las relaciones entre las diferentes variables de interés se realizaron mediante chi cuadrado, correlaciones de Pearson, $t$ de student para muestras independientes, MANOVA y ANOVA de un factor en función de los niveles de la variable criterio. Para medir la fuerza de la asociación de chi cuadrado se emplearon la $C$ de contingencia y la V de Cramer. Los análisis de diferencia de medias, entre victimizados y no victimizados y entre los sujetos que reconocen el maltrato y los que no, en la variable Dependencia Emocional, se realizaron mediante pruebas + para muestras independientes. Los tamaños del efecto se analizaron mediante la d de Cohen, utilizando para su interpretación los criterios propuestos por el autor (Cohen, 1988).

\section{RESULTADOS}

En primer lugar, se utilizó el estadístico Kolgomórov-Smirnov para comprobar el ajuste a la curva normal de la escala Dependencia Afectiva y los macrofactores y factores de interés del IRIDS-100, así como el nivel de significación de los mismos. Los macrofactores Triada Dependiente ( $p=.118$ ) e ldentidad/Caracteriosis ( $p=.054)$ y el factor Búsqueda de Sensaciones ( $p=.118$ ) se ajustaron a la curva normal $y_{1}$ asimismo, se encontró una asimetría positiva para todos ellos al igual que la curtosis, a excepción de esta última en Búsqueda de sensaciones que fue de signo negativo. El mismo proceso se llevó a cabo con los factores de interés del CUVINO-VA, encontrando que ninguno se ajustaba a la curva normal ( $p<.001$ ) y hallando una asimetría y curtosis positiva para todos ellos. Para comprobar la homocedasticidad se empleó la prueba de Levene para la igualdad de varianzas, hallando su cumplimiento en todos los macrofactores y factores salvo en Búsqueda de sensaciones ( $p$ $=.019$ ) y en Humillación Agresor ( $p=.017$ ) Dadas estas condiciones, se justificó la utilización de estadística paramétrica por el elevado tamaño muestral $(N=396)$.

En la Tabla 1 se muestran las frecuencias en victimización psicológica según el criterio de percepción del sujeto sobre su experiencia de

Tabla 1

Frecuencia de victimización por género en función del criterio utilizado

\begin{tabular}{|c|c|c|c|c|}
\hline & Género & Victimización & Frecuencia & $\%$ \\
\hline \multirow{4}{*}{ Tolerancia 0} & \multirow{2}{*}{ Mujer } & Sí & 212 & 80.91 \\
\hline & & No & 50 & 19.08 \\
\hline & \multirow{2}{*}{ Hombre } & Sí & 115 & 85.82 \\
\hline & & No & 19 & 14.18 \\
\hline \multirow{4}{*}{ ¿Te has sentido maltratado/a? } & \multirow{2}{*}{ Mujer } & Sí & 24 & 9.16 \\
\hline & & No & 238 & 90.84 \\
\hline & \multirow{2}{*}{ Hombre } & Sí & 6 & 4.48 \\
\hline & & No & 128 & 95.52 \\
\hline
\end{tabular}


victimización y empleando el criterio de tolerancia cero en función del género. Se constatan diferencias entre ambos criterios, habiendo escasa concordancia $(\kappa=.011, p=.034)$, si bien es cierto que al analizar la relación entre ambos mediante Chi Cuadrado, ésta fue estadísticamente significativa, $\chi^{2}(1)=4.48, p=.034$. A la vista de este resultado y siguiendo las perspectivas teóricas que indican que las víctimas de violencia en la pareja no siempre son capaces de identificarse como tal, se empleó el criterio de tolerancia cero para la división de la muestra en Victimizados-No victimizados para los análisis posteriores. Para la valoración de haber sido agresor o no también se utilizó este último criterio, al no incluir el CUVINOVA una pregunta dicotómica sobre si el sujeto se ha considerado agresor.

A continuación, se presentan los resultados obtenidos en base a los objetivos planteados inicialmente:

1) Relación entre Dependencia emocional y maltrato psicológico

A través de la prueba $t$ de student para muestras independientes, se examinó si la media en la dimensión Dependencia afectiva y los distintos macrofactores y factores de la Triada Dependiente del IRIDS-100 diferían en función de ser victimizado psicológicamente o no. Los sujetos victimizados se distinguieron de los no victimizados en la media en la dimensión Dependencia afectiva, siendo ésta mayor en el caso de los victimizados, lo cual es aplicable a todos los macrofactores y factores asociados (véase Tabla 2). Los tamaños del efecto fueron grandes para Búsqueda de sensaciones ( $d$ $=0.81)$, Autoengaño $(d=1.39)$ y Sentimientos negativos $(d=0.94)$, medios para la dimensión Dependencia afectiva ( $d=0.76)$, el factor Dependencia pura $(d=0.55)$ y los macrofactores Triada dependiente $(d=0.77)$, Acomodación ( $d$ $=0.59)$ e Identidad/Caracteriosis $(d=0.78)$ y pequeño en el caso del factor Craving/Abstinencia $(d=0.42)$.

Cabe destacar que en el caso del factor Búsqueda de sensaciones y en la dimensión Sentimientos negativos, la media de los victimizados fue mayor que el percentil que discrimina entre ausente y presente, siendo éste 2.35 y 2.10 respectivamente, lo cual es indicio de que los sujetos victimizados presentan un nivel patológico medio.

Tabla 2

Diferencias entre victimizados-no victimizados en la dimensión Dependencia afectiva y los macrofactores de Dependencia emocional

\begin{tabular}{|c|c|c|c|c|c|c|}
\hline & \multirow{2}{*}{$\begin{array}{l}\text { Muestra } \\
\text { (V-NO V) }\end{array}$} & \multicolumn{2}{|c|}{ V } & \multicolumn{2}{|c|}{ NO V } & \multirow{2}{*}{ Prueba † } \\
\hline & & $\mathrm{M}$ & $D T$ & $\mathrm{M}$ & $D T$ & \\
\hline Dependencia afectiva & $302-60$ & 2.16 & 0.56 & 1.75 & 0.42 & $\begin{array}{c}t(360)=5.36, p< \\
.001\end{array}$ \\
\hline Triada dependiente & $297-60$ & 2.18 & 0.54 & 1.78 & 0.42 & $\begin{array}{c}t(355)=5.42, p< \\
.001\end{array}$ \\
\hline Dependencia pura & $319-66$ & 1.92 & 0.63 & 1.65 & 0.46 & $\begin{array}{c}t(123.02)=4.06 \\
p<.001\end{array}$ \\
\hline $\begin{array}{l}\text { Búsqueda de } \\
\text { sensaciones }\end{array}$ & $306-66$ & 2.49 & 0.66 & 1.96 & 0.60 & $\begin{array}{c}t(370)=6.00, p< \\
.001\end{array}$ \\
\hline Craving/Abstinencia & $321-66$ & 2.05 & 0.67 & 1.79 & 0.56 & $\begin{array}{c}t(385)=3.12, p= \\
.002\end{array}$ \\
\hline Acomodación & $318-68$ & 1.72 & 0.63 & 1.43 & 0.45 & $\begin{array}{c}t(130.63)=4.41 \\
p<.001\end{array}$ \\
\hline Autoengaño & $301-61$ & 1.94 & 0.62 & 1.43 & 0.28 & $\begin{array}{c}t(196.11)=9.92 \\
p<.001\end{array}$ \\
\hline Sentimientos negativos & $309-65$ & 2.12 & 0.70 & 1.64 & 0.47 & $\begin{array}{c}t(130.30)=6.91 \\
p<.001\end{array}$ \\
\hline Identidad/Caracteriosis & 305-61 & 2.02 & 0.49 & 1.65 & 0.42 & $\begin{array}{c}t(364)=5.60, p< \\
.001\end{array}$ \\
\hline
\end{tabular}


Siguiendo el mismo procedimiento, se examinó si la media en la dimensión Dependencia afectiva y los distintos macrofactores y factores de la Triada dependiente del IRIDS-100 diferían en función de haber agredido psicológicamente o no. Todas las medias de los macrofactores, así como la dimensión Dependencia afectiva difirieron significativamente en función de tratarse de agresores o no agresores, a excepción de en Dependencia pura ( $p=.054)$. En todos los casos la media fue mayor entre los agresores y los tamaños del efecto resultaron medios en Búsqueda de sensaciones $(d=0.57)$, Autoengaño $(d=0.56)$ e ldentidad/Caracteriosis $(d=0.63)$ y pequeños en Dependencia afectiva $(d=0.46)$, Triada dependiente $(d=0.49)$, Craving/Abstinencia ( $d=0.29)$, Acomodación $(d=0.35)$, y Sentimientos negativos $(d=0.49)$. Como ocurrió con la variable victimización, el factor Búsqueda de sensaciones presentó valores medios superiores en los agresores, con puntuaciones mayores que el percentil que discrimina entre ausente y presente y, a su vez, el macrofactor Sentimientos negativos resultó del mismo valor que el percentil (véase Tabla 3).
A objeto de examinar la relación entre la dependencia emocional y la victimización psicológica también se realizó un MANOVA confirmándose valores superiores en todos los indicadores en los sujetos victimizados. Previamente, se verificó que existía relación entre las variables conformantes del constructo dependencia emocional (Bartlett $=0.000, p<$ .001 ) y el cumplimiento de la homocedasticidad univariable en todos los casos, salvo en Autoengaño y Sentimientos negativos, $F(1)=$ 19.69, $p<.001$ y $F(1)=5.48, p=.020$, respectivamente. Los contrastes multivariados revelaron una relación estadísticamente significativa entre las variables dependientes y la victimizació, $F(8)=480.90, p<.001$. A través de las pruebas de los efectos inter-sujetos se observó que las diferencias se debían a la dimensión Dependencia afectiva, así como a todos los macrofactores asociados y los factores de la Triada dependiente, tal y como se expone en la Tabla 4.

El mismo procedimiento se llevó a cabo para la agresión psicológica. La media de los agresores fue más alta que la de los no agresores en todas

Tabla 3

Diferencias entre agresores-no agresores en la dimensión Dependencia afectiva y los macrofactores de Dependencia emocional

\begin{tabular}{|c|c|c|c|c|c|c|}
\hline & \multirow{2}{*}{$\begin{array}{l}\text { Muestra } \\
\text { (V-NO V) }\end{array}$} & \multicolumn{2}{|c|}{ V } & \multicolumn{2}{|c|}{ NO V } & \multirow{2}{*}{ Prueba $\dagger$} \\
\hline & & $M$ & $D T$ & $M$ & $D T$ & \\
\hline Dependencia Afectiva & $300-62$ & 2.13 & 0.56 & 1.88 & 0.52 & $t(360)=3.33, p=.001$ \\
\hline Triada Dependiente & $298-59$ & 2.16 & 0.52 & 1.90 & 0.57 & $t(355)=3.43, p=.001$ \\
\hline Dependencia pura & $322-63$ & 1.90 & 0.62 & 1.73 & 0.59 & $t(383)=1.94, p=.054$ \\
\hline $\begin{array}{l}\text { Búsqueda de } \\
\text { sensaciones }\end{array}$ & $307-65$ & 2.47 & 0.65 & 2.08 & 0.73 & $\mathrm{t}(370)=4.22, \mathrm{p}<.001$ \\
\hline Craving/Abstinencia & $322-65$ & 2.04 & 0.66 & 1.84 & 0.64 & $t(385)=2.20, p=.028$ \\
\hline Acomodación & $320-66$ & 1.70 & 0.63 & 1.49 & 0.51 & $t(384)=2.61, p=.009$ \\
\hline Autoengaño & $303-59$ & 1.91 & 0.60 & 1.57 & 0.56 & $t(360)=3.97, p<.001$ \\
\hline $\begin{array}{l}\text { Sentimientos } \\
\text { Negativos }\end{array}$ & $310-64$ & 2.10 & 0.69 & 1.76 & 0.60 & $t(372)=3.60, p<.001$ \\
\hline $\begin{array}{l}\text { Identidad/ } \\
\text { Caracteriosis }\end{array}$ & $306-60$ & 2.01 & 0.49 & 1.70 & 0.44 & $t(364)=4.50, p<.001$ \\
\hline
\end{tabular}


Tabla 4

Prueba de los efectos inter-sujetos Victimización-dependencia emocional y Agresión-dependencia emocional

\begin{tabular}{|c|c|c|c|cc|}
\hline \multicolumn{6}{|c|}{ Victimización-dependencia emocional Agresión-dependencia emocional } \\
\hline Factores & $\mathrm{F}$ & $\mathrm{p}$ & $\mathrm{R}^{2}$ & $\mathrm{~F} \quad \mathrm{p} \mathrm{R}^{2}$ \\
\hline Dependencia afectiva & 31.70 & $<.001$ & .097 & $18.81<.001 .060$ \\
\hline Triada dependiente & 28.18 & $<.001$ & .087 & $19.14<.001 \quad .061$ \\
\hline Dependencia pura & 9.20 & .003 & .030 & 6.50 .011 .021 \\
\hline Búsqueda de sensaciones & 31.42 & $<.001$ & .096 & $21.56<.001 \quad .068$ \\
\hline Craving/abstinencia & 6.76 & .010 & .022 & $4.31 \quad .039 .014$ \\
\hline Acomodación & 10.07 & .002 & .033 & 5.20 .023 .017 \\
\hline Autoengaño & 38.51 & $<.001$ & .115 & $28.80<.001 .089$ \\
\hline Sentimientos negativos & 25.33 & $<.001$ & .079 & $15.71<.001 \quad .050$ \\
\hline Identidad/Caracterosis & 30.05 & $<.001$ & .092 & $19.42<.001 \quad .062$ \\
\hline
\end{tabular}

las variables. Se comprobó la existencia de relación entre las variables de la dependencia emocional (Bartlett $=0.000, p<.001$ ) y el cumplimiento de la homocedasticidad univariable en todos los casos excepto en Autoengaño, $F(1)=12.95$, $p$ $<.001$, y Sentimientos negativos, $F(1)=5.06, p$ $=.025$. Mediante los contrastes multivariados se observó una relación estadísticamente significativa entre las variables dependientes y la victimización, $F(8)=462.49, p<.001$. Con las pruebas de los efectos inter-sujetos se evaluó qué factores tenían más peso en la diferencia de medias entre agresores y no agresores, encontrando que todos eran significativos (véase Tabla 4).

2) Víctimas de dependencia emocional y maltrato psicológico en función del sexo, la edad, el nivel de estudios y la duración de la relación

A través de análisis de frecuencias y con los percentiles del IRIDS-100, se encontró que, de 116 hombres, el $12.07 \%$ mostraron dependencia afectiva presente, $3.45 \%$ acusada y $13.79 \%$ intensa. En el caso de las 244 mujeres, el 7.79\% mostraron dependencia afectiva presente, $2.46 \%$ acusada y $9.84 \%$ intensa. El resto de la muestra no presentó signos de dependencia afectiva $(70.67 \%$ de los hombres y $79.92 \%$ de las mujeres). En definitiva, un $29.31 \%$ de los hombres y un $20.08 \%$ de las mujeres presentó dependencia afectiva en mayor o menor medida, lo que supuso un $21 \%$ de la muestra total. La media de dependencia afectiva de los hombres fue 2.16, mientras que la de las mujeres fue 2.05, no encontrando diferencias estadísticamente significativas (véase Tabla 5) entre éstas, $t(360)=1.74$, ns. Además, en todos los macrofactores asociados a Dependencia emocional, así como en los factores de la Triada dependiente la media fue más alta entre los hombres, siendo esta diferencia significativa en los macrofactores Triada dependiente, $+(355)=$ 2.26, $p=.024$, Acomodación, $t(384)=4.50, p$ $<.001$, y Autoengaño, $t(360)=2.27, p=.024$, y el factor Dependencia pura, $t(386)=2.63, p=$ .009, aunque con un tamaño del efecto pequeño en todos los casos ( $d=0.25,0.48,0.25$ y 0.28 respectivamente) (véase Tabla 5).

Con respecto al maltrato psicológico, un $82.57 \%$ de la muestra había sido victimizado psicológicamente y un $83.08 \%$ ejerce como agresor de este tipo de violencia. Tal y como se indicó en la Tabla 1, un 85.82\% ( $n=115)$ de los hombres ha sido victimizados psicológicamente y un $88.06 \%$ ( $n=118$ ) ejercen como agresores. A su vez, el $80.92 \%$ ( $n=2012)$ de las mujeres fueron victimizadas y el $80.53 \%(n=211)$ actuaron como agresoras. Mediante la prueba de Chi-Cuadrado se observó que no había relación entre ser hombre o mujer y ser victimizado, $\chi^{2}(1)$ $=1.48$, ns, ni tampoco entre ser hombre o mujer y ejercer como agresor, $\chi^{2}(1)=3.57$, ns.

Para poner en relación la dependencia emocional con las variables sociodemográficas edad y nivel de estudios se utilizó en ANOVA de 
Tabla 5

Presencia e intensidad de dependencia emocional por género

\begin{tabular}{|c|c|c|c|c|c|}
\hline $\begin{array}{l}\text { Dependencia } \\
\text { emocional }\end{array}$ & Hombres \% & Mujeres \% & $\begin{array}{l}\text { Dimensiones } \\
\text { Dependencia } \\
\text { emocional }\end{array}$ & Prueba $t$ & $d$ \\
\hline Presente & 12.07 & 7.79 & $\begin{array}{c}\text { Triada } \\
\text { dependiente }\end{array}$ & $\begin{array}{c}(\mathrm{t}(355)=2.26, \mathrm{p} \\
=.024)\end{array}$ & 0.25 \\
\hline Acusada & 3.45 & 2.46 & Acomodación & $\begin{array}{c}(t(384)=4.50, p \\
<.000)\end{array}$ & 0.48 \\
\hline Intensa & 13.79 & 9.84 & Autoengaño & $\begin{array}{c}(t(360)=2.27, p \\
=.024)\end{array}$ & 0.25 \\
\hline Ausente & 70.77 & 79.92 & $\begin{array}{l}\text { Dependencia } \\
\text { pura }\end{array}$ & $\begin{array}{c}(t(386)=2.63, \mathrm{p} \\
=.009)\end{array}$ & 0.28 \\
\hline Media D.E. & 2.16 & 2.05 & & & \\
\hline
\end{tabular}

un factor. De este modo, se halló que no existe relación entre la dimensión Dependencia afectiva y en ninguno de los macrofactores y factores asociados con el nivel de estudios a excepción del macrofactor Sentimientos negativos, Welch(3) $=2.88, p=.042$, observando que la diferencia significativa se concentra entre los estudiantes de Educación Secundaria Obligatoria y los universitarios, siendo la media de estos últimos significativamente más alta. Tampoco se encontró asociación entre éstos y la edad considerada en rangos. Para valorar esta misma relación pero con la edad como variable cuantitativa se realizaron correlaciones de Pearson, resultando únicamente significativa la que se da entre la edad y el macrofactor Identidad/Caracterosis, $r(365)=.123, p=.018$.

Para evaluar el maltrato psicológico en función de la edad, se empleó la prueba † de Student, hallándose diferencias significativas, $t(394)=2.08, p=.038$, entre victimizados $(M$ $=19.48, D T=2.58)$ y no victimizados $(M=$ $18.77, D T=2.62$ ) aunque con un tamaño del efecto pequeño $(d=0.27)$. Esto se confirmó también en el caso de la agresión, t(394) = 2.22, $p=.027$, con una media de edad más alta entre los agresores $(M=19.49, D T=2.58)$ que en los no agresores $(M=18.72, D T=2.59)$ con un tamaño del efecto pequeño $(d=0.30)$.

En cuanto a la victimización de maltrato psicológico, a través del estadístico ChiCuadrado, ésta se relacionó con el nivel de estudios, $\chi^{2}(3)=10.61, p=.014$, aunque con una asociación no demasiado elevada $(\mathrm{V}=$
.16), observándose una diferencia significativa en el nivel de estudios Educación Secundaria Obligatoria, habiendo un destacable menor número de casos de victimización en este grupo. La misma relación se encontró con respecto a la agresión, $\chi^{2}(3)=9.25, p=.026$, con una fuerza de asociación pequeña $(V=.15)$, hallando que entre los estudiantes de ESO había un número significativamente menor de agresores.

Por último, a través de la prueba t de Student para muestras independientes se valoró la relación tanto de la victimización psicológica como de la agresión con la duración de la relación, llegando a la conclusión de que no se relaciona en ninguno de los dos casos, $t(126.766)=0.43$, ns y $t(128.75)=1.13, n s$, respectivamente.

3) Identificación de los victimizados como maltratados

En primer lugar, se valoró si ser víctima de violencia psicológica guardaba relación con ser agresor de esta misma, hallando que existe una asociación entre ambas variables, $\chi^{2}(1)=245.32, p<.001$, siendo el efecto de ésta bastante grande $(C=.62)$. En el grupo de victimizados se encontró una frecuencia de agresores mayor de la esperada, del mismo modo que en el de no victimizados había menos agresores de los esperados.

De los 327 sujetos victimizados, tan solo un $8.87 \%$ (el $5.22 \%$ de los hombres y el $10.85 \%$ de las mujeres) se consideró maltratado 
(respuesta afirmativa al ítem dicotómico "¿̇Te has sentido maltratado/a?"); sin embargo, se encontró una relación significativa entre ser víctima y reconocerse como tal, $\chi^{2}(1)=4.48$, $p=.034$. Considerando dos grupos, los que se identifican como maltratados y los que no, se encontraron diferencias significativas entre las medias de la dimensión Dependencia afectiva, $t(300)=5.87, p<.001$, de los macrofactores Triada dependiente, $t(295)=$ 4.52, $p<.001$, Acomodación, $+(28.180)=$ 3.65, $p<.001$, Autoengaño, $+(299)=6.30$, $p<.001$, Sentimientos negativos, $t(307)=$ 6.61, $p<.001$, e Identidad/Caracteriosis, $t(303)=3.96, p<.001$, y de factores de interés Dependencia pura, $t(317)=5.51, p<.001$, Búsqueda de sensaciones, $+(304)=2.76, p=$ .006, y Craving/Abstinencia, $+(319)=2.66$, $p=.008$, observando medias más altas para aquellos sujetos que reconocen haber sufrido maltrato. Los tamaños del efecto fueron grandes para la dimensión Dependencia afectiva $(d=$ 1.18), los macrofactores Triada dependiente (d $=0.89$ ), Autoengaño ( $d=1.23)$, y Sentimientos negativos $(d=1.35)$ y el factor Dependencia pura $(d=1.07)$ y medios para Acomodación ( $d$ $=0.73)$, Identidad/Caracteriosis $(d=0.79)$ y los factores Búsqueda de sensaciones y Craving/ Abstinencia ( $d=0.53$ ambos).

\section{DISCUSIÓN}

Tras evaluar la relación entre la dependencia emocional y el maltrato psicológico bien como víctima, bien como agresor, en respuesta a los objetivos planteados en el estudio, cabría destacar los siguientes hallazgos: 1) existe relación entre dependencia emocional y maltrato psicológico, ésta se da tanto en victimización como en agresión; 2) un $21 \%$ de la muestra presenta dependencia emocional, encontrándose más hombres (29.31\%) que mujeres $(20.08 \%)$ con esta patología. Un $82.57 \%$ ha sido victimizado psicológicamente (un $85.82 \%$ de los hombres y un $80.92 \%$ de las mujeres) y un $83.08 \%$ ha sido agresor de este tipo de violencia (un $88.06 \%$ de los hombres y un $80.53 \%$ de las mujeres); 3) a nivel general, la dependencia emocional no se relaciona con el género (aunque sí lo hacen los macrofactores Triada dependiente, Acomodación, Autoengaño y el factor Dependencia pura), la edad (a excepción del macrofactor Identidad/Caracterosis) y el nivel de estudios (salvo con el macrofactor Sentimientos negativos), mientras que en el maltrato psicológico no se hallaron diferencias en función del género y de la duración de la relación, pero sí de la edad; 4) existe relación entre la dependencia emocional y la conciencia de maltrato, con niveles de dependencia más alta en aquellos sujetos victimizados que se reconocen como tal.

Como se esperaba, se encontró relación entre la dependencia emocional y el maltrato psicológico, al igual que comprobaron Momeñe, Jauregui y Estévez (2017). Si bien es cierto que la diferencia de medias entre victimizados y no victimizados se confirmó tanto en la escala Dependencia Afectiva como en todos los macrofactores y factores asociados, se obtuvo un tamaño del efecto grande en el factor Búsqueda de sensaciones y en los macrofactores Autoengaño y Sentimientos negativos. Mediante el MANOVA se corroboró que el macrofactor Autoengaño es el que más peso presenta en la diferencia de medias. La elevada puntuación en el factor Búsqueda de sensaciones podría explicarse por la edad de la muestra, ya que se ha observado que la búsqueda de sensaciones es más prevalente en el rango de edad 20-29 años (Chico-Librán, 2000), similar al empleado en este estudio.

Por lo que respecta a la agresión psicológica, también se obtuvieron diferencias significativas en las medias de los agresores y los no agresores, con elevados tamaños del efecto en Autoengaño, Identidad/Caracteriosis y Búsqueda de sensaciones. La importancia del factor Búsqueda de sensaciones, al igual que en la victimización, se podría explicar por la edad (Moral et al., 2017). La importancia del factor Identidad/Caracterosis en agresores encaja con el control, dominio, sometimiento y poder mediante los que se les describe (Moral y Sirvent, 2008), así como por el propio rol de los agresores de violencia en la pareja. A su vez, la puntuación más alta en Autoengaño apoya el reciente estudio de Vecina (2018) que propone éste como un mecanismo para mantener la integridad moral. A la vista de estos resultados cabe destacar que 
el Autoengaño, como constructo que induce a un registro distorsionado de la realidad tanto en víctimas como en agresores, es el factor que mejor explica la relación existente entre dependencia emocional y maltrato psicológico, lo cual coincide con resultados previos (Moral et al., 2018).

En los estudios sobre dependencia afectiva y género (Moral y Sirvent, 2009) se sostiene que los varones son más buscadores de sensaciones y más hiperestimuladores, mientras que las mujeres puntuarían más alto en Autoengaño. Sin embargo, los resultados del presente estudio muestran una media más elevada en todos los macrofactores para los hombres y una mayor presencia de dependientes emocionales en este grupo. Este hallazgo coincide con los datos obtenidos en el estudio de González-Jiménez y Hernández-Romera (2014), en el que se observó una mayor prevalencia de varones con dependencia emocional, aunque no encontraron diferencias estadísticamente significativas con respecto al género. Estos resultados contradicen la preconcebida idea popular de que la dependencia emocional es más prevalente en las mujeres.

Por lo que respecta al maltrato psicológico, en la mayor parte de las investigaciones en relaciones heterosexuales, se muestran a las mujeres como víctimas y a los hombres como agresores. No obstante, en este estudio los hombres presentaron una mayor prevalencia de victimización, aunque la relación entre victimización y género no fue significativa. Este hecho puede tener dos posibles explicaciones. Por una parte, se podría aludir a la citada bidireccionalidad de la violencia, según la cual en las parejas en las que se da maltrato serían agresor y víctima ambos miembros. Por otra parte, esta ausencia de diferencias intergénero puede estar influenciada por el tipo de violencia que se estudia, ya que se ha observado que las mujeres perpetran el abuso psicológico con más frecuencia que los hombres, que más comúnmente suelen ejercer violencia física o sexual (Batiza, 2017). En muchos de los estudios que consideran a las mujeres como víctimas se analiza la violencia en la pareja en su conjunto y, por tanto, al tener en cuenta también el maltrato físico y el sexual, se podrían distorsionar los resultados (aumento del número de mujeres como víctimas y de hombres como agresores).

En base a la literatura, también se propuso que el mayor número de casos de victimización se daría en torno a los veinte años (véase Johnson, Giordano, Manning y Longmore, 2015). Al medir la edad por rangos no se encontró ninguna relación, a pesar de que se esperaba que el mayor número de casos de victimización se diera en el rango de 19 a 21 años. Por el contrario, al tener en cuenta la edad como una variable continua, se encontró una diferencia de medias entre victimizados y no victimizados, estando la de los primeros muy cercana a los veinte, mientras que la edad de los no victimizados era menor. Este hallazgo encaja a la vez con el resultado de que los estudiantes de Secundaria presentan una victimización significativamente menor que los universitarios. Sin embargo, es contradictorio con los estudios de Redondo, Inglés y García (2017) en los que se encontró una mayor tasa de victimización entre los sujetos de menor edad. Los mismos resultados en cuanto a la edad se hallaron entre los agresores y los no agresores, lo que se puede deber en parte de nuevo a la bidireccionalidad. En cualquier caso, cabe destacar que efectivamente tanto los sujetos victimizados como los agresores tenían una media de edad más cercana a los 20 años.

Por último, se hipotetizó que de los sujetos victimizados un gran porcentaje no se identificaría como maltratado. Efectivamente así fue, ya que solo un $8.87 \%$ se consideró víctima de malos tratos, dato que supera el índice de aproximadamente 6\% encontrado por RodríguezFranco et al. (2012).

Otro objetivo del presente estudio consistió en analizar la relación entre la dependencia emocional y la conciencia de maltrato. Se confirmó que la dependencia emocional está más presente entre los sujetos que se reconocían como víctimas respecto a los que no, confirmándose en la escala de Dependencia Afectiva, así como en los macrofactores Triada Dependiente, Autoengaño, Sentimientos Negativos y el factor Dependencia Pura. Este resultado es contradictorio teniendo en cuenta las características del dependiente emocional, sobre todo en lo relativo al constructo Autoengaño que ha demostrado ser relevante en la relación dependencia emocional-maltrato psicológico (Moral et al., 2018). 
Este estudio presenta una serie de limitaciones que cabe mencionar. En primer lugar, el uso del criterio tolerancia cero supone que no se valoren niveles de gravedad de la victimización y, por lo tanto, no se pueda establecer una relación dependencia emocionalintensidad de la victimización. Además, al ser el maltrato psicológico el más prevalente de los tipos de violencia en el noviazgo y considerarse victimizado a todo sujeto que responda afirmativamente a un ítem, el número de victimizados fue mucho mayor que el de no victimizados (327 vs. 69), lo cual puede estar sobreestimado. En relación con el CUVINOVA también cabe destacar que, aunque en los análisis solo se tuvieron en cuenta los factores referentes a violencia psicológica (Humillación, Desapego y Coerción), los sujetos respondieron al cuestionario completo y se pudo dar el caso de que alguno se reconociese como maltratado, pero el tipo de victimización sufrida fuera física - sexual. Es pertinente señalar también el carácter transversal del estudio, que dificulta establecer el sentido de la causalidad entre las variables estudiadas. Por último, debemos ser precavidos en la interpretación de los resultados, pues la extensión total de los cuestionarios aplicados pudo haber provocado fatiga en los participantes. Además, las respuestas de los participantes es posible que estén sujetas a un sesgo de respuestas, disimulación, entendida como la asunción de una imagen positiva por deseabilidad social (Impresión Management antes mencionada) (Fariña, Redondo, Seijo, Novo, y Arce, 2017).

A la vista de todo lo expuesto se hace precisa una mayor educación afectiva, mediante la que se desmitifique el concepto de amor romántico y se enseñe desde la infancia a establecer relaciones sanas no sexistas y, sobre todo, a diferenciar éstas de las que no lo son. En este sentido, se considera sumamente relevante promover investigaciones sobre la problemática de la dependencia emocional y las actitudes sexistas, dada su trascendencia en las manifestaciones de violencia en el noviazgo y de maltrato psicológico en particular (Bringas et al., 2017; García el al., 2018; Moral et al, 2017; Valle y Moral, 2018). Dada la multicausalidad de la problemática investigada se precisa promover una evaluación integral, considerándose la dependencia emocional como uno de los posibles factores determinantes.

\section{- Conflicto de intereses}

Los autores declaran no tener ningún conflicto de intereses.

\section{REFERENCIAS}

Amor, P., y Echeburúa, E. (2010). Claves psicosociales para la permanencia de la víctima en una relación de maltrato. Clínica Contemporánea, 1(2), 97-104. https://dx.doi. org/10.5093/cc2010v1n2a3

Arce, R., Fariña, F., Seijo, D., y Novo, M. (2015). Assessing impression management with the MMPI-2 in child custody litigation. Assessment, 22(6), 769-777. http://dx.doi. org/10.1177/1073191114558111

Barrón, A., De Paúl, P., Martínez-lñigo, D., y Yela, C. (1999). Beliefs and romantic myths in Spain. The Spanish Journal of Psychology, 2(1), 64-73. https://dx.doi.org/10.1017/ $\underline{51138741600005461}$

Batiza, F. J. (2017). La violencia de pareja: Un enemigo silencioso. Archivos de Criminología, Seguridad Privada y Criminalística, 18, 144151. Recuperado de https://dialnet.unirioja. es/ejemplar/450108

Bonomi, A. E., Anderson, M. L., Nemeth, J., Bartle-Haring, S., Buettner, C., y Schipper, D. (2012). Dating violence victimization across the teen years: abuse frequency, number of abusive partners, and age at first occurrence. BMC Public Health, 12, 2-10. https://doi. org/10.1186/1471-2458-12-637

Bringas-Molleda, C., Estrada-Pineda, C., SuárezÁlvarez, J., Torres, A., Rodríguez-Díaz, F. J., García-Cueto, E., y Rodríguez-Franco, L. (2017). Actitud sexista y trascendente durante el noviazgo entre universitarios latinoamericanos. Revista Iberoamericana de Psicología y Salud, 8(1), 44-55. https://doi.org/10.23923/i. rips.2017.08.005

Celis, A., y Rojas, J. (2015). Violencia en el noviazgo desde la perspectiva de varones adolescentes. Informes Psicológicos, 15, 83-104. https:// dx.doi.org/10.18566/infpsicv15n1a05 
Chico-Librán, E. (2000). Búsqueda de sensaciones. Psicothema, 12(2), 229-235. Recuperado de https://www.psicothema.es/pdf/282.pdf

Cohen, J. W. (1988). Statistical power analysis for the behavioral sciences (2a. ed). Hillsdale, NJ: LEA.

Estévez, A., Urbiola, I., Iruarrizaga I., Onaindia, J., y Jauregui, P. (2017). Emotional dependency in dating relationships and psychological consequences of internet and mobile abuse. Anales de Psicología, 33(2), 260-268. https:// dx.doi.org/10.6018/analesps.33.2.255111

Fariña, F., Redondo, L., Seijo, D., Novo, M., y Arce, R. (2017). A meta-analytic review of the MMPI validity scales and indexes to detect defensiveness in custody evaluations. International Journal of Clinical and Health Psychology, 17, 128-138. http://dx.doi. org/10.1016/i.ijchp.2017.02.002

García, V., Lana, A., Fernández, A., Bringas, C., Rodríguez-Franco, L., y Rodríguez-Díaz, F. J. (2018). Actitudes sexistas y reconocimiento del maltrato en parejas jóvenes. Atención Primaria, 50(7), 398-405. https://dx.doi.org/10.1016/i. aprim.2017.04.001

Gil-Olarte, P., Cavalcante, A., Paramio, A., Zayas, A., y Guil, R. (2017). Impulsividad y búsqueda de sensaciones: implicaciones de intervención en jóvenes. International Journal of Developmental and Educational Psychology. INFAD: Revista de Psicología, 1(2), 393-404. https://doi. org/10.17060/ijodaep.2017.n1.v2.952

González-Jiménez, A. J., y Hernández-Romera, M. D. (2014). Emotional dependency based on the gender of young adolescents in Almeria, Spain. Procedia: Social and Behavioral Sciences, 132, 527-532. https://doi.org/10.1016/i. sbspro.2014.04.348

Jennings, W. G., Okeemb, C., Piquero, A. R., Sellers, C. S., Theobald, D., y Farrington, D. P. (2017). Dating and intimate partner violence among young persons ages 15-30: Evidence from a systematic review. Aggression and Violent Behavior, 33, 107-125. https://doi. org/10.1016/i.avb.2017.01.007

Johnson, W. L., Giordano, P. C., Manning, W. D., y Longmore, M. A. (2015). The age-IPV curve: changes in the perpetration of intimate partner violence during adolescence and young adulthood. Journal of Youth and Adolescence, 44(3), 708-726. https://dx.doi.org/10.1007/ s10964-014-0158-z

Kemer, G., Yildiz, E. C., y Bulgan, G. (2016). Emotional dependency and dysfunctional relationship beliefs as predictors of married Turkish individuals' relationship satisfaction. Spanish Journal of Psychology, 19, 1-8. https:// dx.doi.org/10.1017/sip.2016.78

Lemos-Hoyos, M., Jaller-Jaramillo, C., GonzálezCalle, A. M., Díaz-León, Z. T., y De la Ossa, D. (2012). Perfil cognitivo de la dependencia emocional en estudiantes universitarios en Medellín, Colombia. Universitas Psychologica, 11 (2), 395-404. Recuperado de http://www. scielo.org.co/pdf/rups/v1 1 n2/v 11 n2a04.pdf

López-Cepero, J., Rodríguez-Franco, L., RodríguezDíaz, F. J., Bringas, C., y Paíno, S. (2015). Percepción de la victimización en el noviazgo de adolescentes y jóvenes españoles. Revista Iberoamericana de Psicología y Salud, 6, 64-71. https://doi.org/10.1016/i.rips.2015.04.001

Medrano, A., Miranda, M., y Figueras, V. M. (2017). Violencia de pareja contra las mujeres en México: Una mirada a la atención del sector salud desde una perspectiva interseccional. Multidisciplinary Journal of Gender Studies, 6, 1231-1262. http://dx.doi.org/10.17583/ generos.2017.2131

Momeñe, J., Jáuregui, P., y Estévez, A. (2017). El papel predictor del abuso psicológico y la regulación emocional en la dependencia emocional. Behavioral Psychology/Psicología Conductual, 25(1), 65-78.

Moral, M. V., y Sirvent, C. (2008). Dependencias sentimentales $\mathrm{a}$ afectivas: etiología, clasificación y evaluación. Revista Española de Drogodependencias, 33 (2), 150-167. Recuperado de https://www.aesed.com/ upload/files/vol-33/n-2/v33n2_2.pdf

Moral, M. V., y Sirvent, C. (2009). Dependencia afectiva y género: Perfil sintomático diferencial en una muestra de dependientes afectivos españoles. Interamerican Journal of Psychology, 43(2), 230-240. Recuperado de https://www. redalyc.org/articulo.oa? id $=28412891004$

Moral, M. V., García, A., Cuetos. G., y Sirvent, C. 
(2017). Violencia en el noviazgo, dependencia emocional y autoestima en adolescentes y jóvenes españoles. Revista Iberoamericana de Psicología y Salud, 8(2), 96-107. https:// dx.doi.org/10.23923/i.rips.2017.08.009

Moral, M. V., Sirvent, C., y Ovejero, A. (2018). Dependencia emocional en las relaciones de pareja como síndrome de Artemisa: modelo explicativo. Terapia Psicológica, 36(3), 156 166. Recuperado de: https://teps.cl/index. php/teps/article/view/240

Muñoz-Rivas, M. J., Graña, J. L., y González, M. P. (2011). Abuso psicológico en parejas jóvenes. Behavioral Psychology/Psicología Conductual, 19(1), 117-131.

Palmetto, N., Davidson, L. L., Breitbart, V., y Rickert, V. I. (2013). Predictors of physical intimate partner violence in the lives of young women: Victimization, perpetration, and bidirectional violence. Violence and Victims, 28, 103-121. https://doi:10.1891/0886-6708.28.1.103

Petruccelli, F., Diotaiuti, P., Verrastro, V., Petruccelli, I., Federico, R., Martinotti, G., Fossati, A., Di Giannantonio, M., y Janiri, L. (2014). Affective dependence and aggression: An Exploratory study. Biomed Research International, 1-11. https://dx.doi.org/10.1155/2014/805469

Redding, E. M., Ruiz, M. T., Fernández, J., y Guijarro, M. (2017). Gender inequality and violence against women in Spain, 2006-2014: Towards a civilized society. Gaceta Sanitaria, 31, 82-88. https://dx.doi.org/10.1016/i. gaceta.2016.07.025

Redondo, J., Inglés, C. J., y García, K. (2017). Papel que juega la edad en el noviazgo de estudiantes de la Universidad Pontificia Bolivariana de Bucaramanga. Diversitas: Perspectivas en Psicología, 13 (1), 41 - 54. Recuperado de https://www.scielo.org.co/pdf/ dpp/v13n1/1794-9998-dpp-13-01-00041. pdf

Rodríguez-Díaz, J., Herrero, J., Rodríguez-Franco, L., Bringas-Molleda, C., Paíno-Quesada, S., y Pérez, B. (2017). Validation of Dating Violence Questionnarie-R (DVQ-R). International Journal of Clinical and Health Psychology, 17(1), 77-84. https://dx.doi.org/10.1016/i. ïchp.2016.09.001
Rodríguez-Franco, L., Antuña-Bellerín, M. A., López-Cepero, J., Rodríguez-Díaz, F. J., y Bringas, C. (2012). Tolerance towards dating violence in Spanish adolescents. Psicothema, 24 (2), 236-242. Recuperado de http://www. psicothema.com/pdf/4005.pdf

Rubio-Garay, M., López-González, M. A., Carrasco, M. A., y Amor, P. J. (2017). Prevalencia de violencia en el noviazgo: una revisión sistemática. Papeles del Psicólogo / Psychologist Papers, 38 (2), 135-147. https:// dx.doi.org/10.23923/pap.psicol2017.2831

Rubio-Garay, F., López-González, M. A., Saúl, L. A., y Sánchez-Elvira-Paniagua, A. (2012). Direccionalidad y expresión de la violencia en las relaciones de noviazgo de los jóvenes. Acción Psicológica, 9 (1), 61 -70. https://dx.doi. org/10.5944/ap.9.1.437

Sirvent, C., y Moral, M. V. (2018). Construcción y validación del inventario de relaciones interpersonales y dependencias sentimentales (IRIDS-100). Health and Addictions, 18(2), 35-47. hittps://dx.doi.org/10.21134/haaj. v18i2.342

Sirvent, C., Herrero, J., Moral, M. V., y Rodríguez, F. J. (2019). Evaluation of self-deception: factorial structure, reliability and validity of the SDQ-12 (Self-deception Questionnaire). Plos One, 14(1), e0210815. https://dx.doi. org/10.1371/journal.pone.0210815

Siödin, A. K., Wallinius, M., Billstedt, E., Hofvander, B., y Nilsson, T. (2017). Dating violence compared to other types of violence: Similar offenders but different victims. European Journal of Psychology Applied to Legal Context, 9(2), 83-91. https://doi.org/10.1016/i. ejpal.2017.03.001

Temple, J. R., Choi, H. J., Elmquist, J., Hecht, M., Miller-Day, M., Stuart, G. L., Brem, M., y Wolford-Clevenger, C. (2016). Psychological abuse, mental health, and acceptance of dating violence among adolescents. Journal of Interpersonal Violence, 59(2), 197-202. https://dx.doi.org/10.1016/i. jadohealth.2016.03.034

Urbiola, I., y Estévez, A. (2015). Emotional dependency and early maladaptive schemas in adolescents and youth dating relationships. 
Behavioral Psychology / Psicología Conductual, 23 (3), 571-587. Recuperado de https://www. redalyc.org/html/167/16750533007/

Urbiola, I., Estévez, A., Iruarrizaga, I., y Jauregui, P. (2017). Emotional dependency in youth: Relationship with anxious and depressive symptoms, self-esteem and gender differences. Ansiedad y Estrés / Anxiety and Stress, 23(1), 6-11. https://dx.doi.org/10.1016/i. anyes.2016.11.003

Valle, L., y Moral, M. V. (2018). Dependencia emocional y estilo de apego adulto en las relaciones de noviazgo en jóvenes españoles. Revista Iberoamericana de Psicología y Salud, 9(1), 27-41. https://doi.org/10.23923/i. rips.2018.01.013

Vecina, M. L. (2018). How can men convicted of violence against women feel moral while holding sexist and violent attitudes? A homeostatic moral model based on self-deception. American Journal of Men's Health, 1-9. https://dx.doi. org/10.1177/1557988318774218

Zamora-Damián, G., Alvídrez, S., Aizpitarte, A., y Rojas-Solís, J. L. (2018). Prevalencia de violencia en el noviazgo en una muestra de varones adolescentes mexicanos. Revista de Psicología y Ciencias del Comportamiento de la Unidad Académica de Ciencias Jurídicas y Sociales, 9, 30-53. Recuperado de https://www. aacademica.org/dr.jose.lvis.rojas.solis/32.pdf 